\title{
ASYMPTOTIC NUMERICAL METHOD AND POLYNOMIAL CHAOS EXPANSION FOR THE STUDY OF STOCHASTIC NON-LINEAR NORMAL MODES
}

\author{
Alfonso M. Panunzio \\ Laboratoire MSSMat \\ École Centrale de Paris \\ Châtenay-Malabry, 92290, France \\ Email: alfonso.panunzio@ecp.fr \\ Christoph Schwingshackl \\ Vibration University Technology Centre \\ Imperial College London \\ London, SW72AZ, United Kingdom \\ Email: c.schwingshackl@imperial.ac.uk
}

\author{
Loïc Salles * \\ Vibration University Technology Centre \\ Imperial College London \\ London, SW72AZ, United Kingdom \\ Email: I.salles@imperial.ac.uk \\ Muzio Gola \\ Department of Aerospace engineering \\ Politecnico di Torino \\ Turin, 10129, Italy \\ Email: muzio.gola@polito.it
}

\begin{abstract}
Nonlinear normal mode (NNM) analysis is one emerging technique to analyse the nonlinear vibration of bladed-disk. It links the resonance frequency to the energy present in the system, and allows a simple identification of internal resonances in the structure. Non-linear vibration analysis is traditionally carried out under the assumption that the mechanical properties and forcing function are deterministic. Since every mechanical system is by nature uncertain a truly accurate nonlinear dynamic analysis requires the inclusion of random variables in the response predictions. The propagation of random input uncertainties in a NNM analysis is the main aim of the presented work. The Asymptotic Numerical Method (ANM) will be used to calculate the NNMs for a contact problem in a computationally efficient way. The stochastic NNM permits to quantify the effect of uncertainties on the resonance frequency and the change in mode shape due to non-linearities, leading to the calculation of uncertain internal resonances. The proposed method is initially applied to a simple spring-mass system to demonstrate the effects of uncertainty on the NNM predictions. In a second step a blade-casing interaction with localized contact non-linearity is investigated with a real geometry. The resulting NNMs show the presence of internal resonance for both cases.
\end{abstract}

\footnotetext{
*Address all correspondence to this author.
}

\section{NOMENCLATURE}

$\omega, \eta \quad$ frequency,regularization parameter

$f, k_{n l} \quad$ nonlinear force and contact stiffness

$\boldsymbol{M}, \boldsymbol{K}, \boldsymbol{G}$ mass, stiffness matrices and implicit quadratic function

$\xi$ random variable

$\Psi_{k} \quad$ Polynomial chaos basis function

$\beta_{k} \quad k$-th stochastic mode

ANM Asymptotic Numerical Method.

DOF Degree Of Freedom.

HBM Harmonic Balance Method.

NNM Non-linear Normal Mode.

PCE Polynomial Chaos Expansion.

\section{INTRODUCTION}

One of the major drivers of aeroengine design is an increase in the efficiency of the engine. One of the possibilities to increase 
the aerodynamic efficiency of the aircraft engine is a minimisation of the tip clearance between the blade and the casing, leading to an increased probability of blade casing interaction. Manufacturers need to be able to predict the risk of blade casing interaction early on during the design to better understand the vibration behavior of the bladed-disk during a contact interaction. A range of studies is available to understand the physics of the blade casing interaction numerically [1,2] and experimentally[3, 4].

One approach to study the interaction of blades with casings is the concept of non-linear modes (NNM). The concept of a nonlinear normal mode has been introduced by Rosenberg in the 1960s [5]. Rosenberg defines the NNM as a "vibration in unison" of the system. Other definitions of the NNM have been proposed by different authors [6, 7]. More recently Kerschen et al. [8] have presented a full framework for the Nonlinear Normal Mode Concept. Laxalde [9] has recently studied the blade-casing interaction using the NNM concept and demonstrated feasibility of the approach.

The uncertainties play an important role in blade casing interaction especially since the initial gap between the blade tip and the casing is uncertain due to the tolerance in the manufacturing, inaccuracy in the assembly, and the aerodynamic loading. In order to be predictive and robust the uncertainties have to be introduce in the system.

This study will extend the application of NNMs for blade casing interaction to include uncertain behaviour in the analysis. Polynomial Chaos Expansion (PCE) will be used to quantify the uncertainties of the response (NNM). Didier et al. [10] have successfully applied the PCE with the harmonic balance method to stochastic frequency responses of nonlinear system. Their work is based on an intrusive Galerkin approach of the PCE. In this paper we propose to use a non-intrusive approach coupled with a harmonic balance method and the Asymptotic Numerical approach [11] for the calculation of the uncertain NNM. Using the non-intrusive approach for the calculation of PCE minimises the requirements of coding and allows an easy coupling of the PCE to the NNM analysis for blade casing interaction. The developed approach will be demonstrated with a basic 2 DOF system and a full 3D blade interaction with a rigid casing.

\section{CALCULATION OF NON-LINEAR NORMAL MODES}

The calculation of non-linear normal modes (NNM) is based on the strategy proposed by Moussi [11], which has been implemented in the open source FE solver Code-Aster for general use. What follows is a short summary of the principle to calculate NNMs with the help of the harmonic balance method (HBM) and the asymptotic numerical approach (ANM), to help with the understanding of its novel implementation with the polynomial chaos expansion (PCE) for the calculation of uncertain NNMs.

\section{Equation of autonomous system with unilateral con- tact}

The equation of motion of an autonomous nonlinear dynamical system can be written as:

$$
\boldsymbol{M} \ddot{\boldsymbol{U}}+\boldsymbol{K} \boldsymbol{U}+\boldsymbol{F}_{N L}(\boldsymbol{U})=\mathbf{0}
$$

where $\boldsymbol{M}$ is the mass matrix, $\boldsymbol{K}$ is the stiffness matrix, $\boldsymbol{F}_{N L}$ is the nonlinear function and $\boldsymbol{U}$ is the displacement vector. It is assumed, that the contact forces due to the blade casing interaction are localized and depend on the relative displacement $u_{c}(t)$ between the flexible blade and a rigid casing. The contact law can then be expressed in the following form [11]:

$$
f_{n l}\left(u_{c}(t)\right)=\left\{\begin{array}{l}
k_{n l}\left(u_{c}(t)-g\right) \text { if } u_{c}(t) \geq g \\
0 \text { if } u_{c}(t)<g
\end{array}\right.
$$

where $k_{n l}$ is the contact stiffness, and $g$ is the initial gap between the node of the structure and the obstacle. In order to solve the dynamic contact problem with the asymptotic numerical [12] method Eqn. 2 has to be regularized and rewritten in a quadratic form [11]:

$$
f(t)\left(f(t)-k_{n l}\left(u_{c}(t)-g\right)\right)-k_{n l} \eta=0
$$

where $\eta$ is the regularization parameter. The contact force is not modelled as a quadratic nonlinearity but is defined implicitly by the quadratic equation Eq. 3, which permits to apply the asymptotic numerical method. The expression of the regularized nonlinear force is introduced in the equation of motion Eqn.1 leading to new unknowns: the nonlinear forces $f$. The resulting quadratic equations of motion of the contact problem now become

$$
\begin{aligned}
\boldsymbol{M} \ddot{\boldsymbol{U}}+\boldsymbol{K} \boldsymbol{U}+\boldsymbol{F} & =\mathbf{0} \\
\boldsymbol{G}(\boldsymbol{F}, \boldsymbol{U}) & =\mathbf{0}
\end{aligned}
$$

where $\boldsymbol{G}$ is the implicit function defining all the nodal contact forces from Eqn.(3) and $\boldsymbol{U}, \boldsymbol{F}$ are respectively the vector of displacements and contact forces. The vector of unknowns is rewritten as $\boldsymbol{X}=\{\boldsymbol{U}, \boldsymbol{F}\}^{T}$ for which a solution must be found. The contact forces are shown on Fig. 1 for different values of $\eta$ with $k_{n l}=40 e 6$ and $g=1 e-5$. The value $\eta=5 e-6$ permits a good accuracy with a well conditioned problem. This value was used in the simulations. 


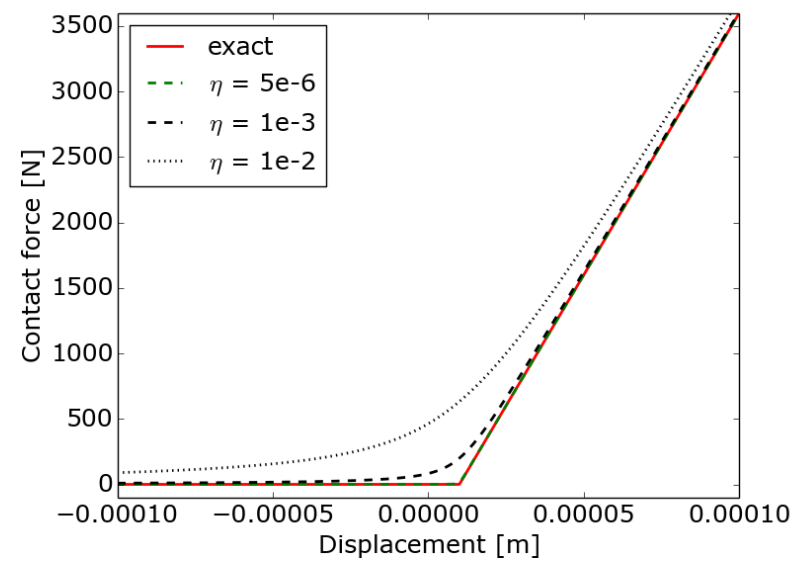

FIGURE 1. NONLINEAR FORCES VERSUS THE DISPLACEMENT FOR DIFFERENT REGULARIZATION PARAMETER $\eta$.

\section{Harmonic Balance and Asymptotic Numerical Method for NNM}

In order to find a solution for the the NNMs in quadratic form, the harmonic balance method is combined with the asymptotic numerical method. Assuming periodicity, the nonlinear response is approximated by a truncated Fourier series. In order to improve the computation time of the HBM, Moussi [11] proposed an approach with a different number of harmonics for the displacements and the forces. In order to describe the nonlinear forces accurately a high harmonic order $H_{f}$ is necessary while a smaller order $H_{u}$ is sufficient for the displacements.

$$
\begin{aligned}
& \boldsymbol{u}(t)=\boldsymbol{U}^{0}+\sum_{n=1}^{H_{u}} \boldsymbol{U}^{n, c} \cos (n \omega t)+\boldsymbol{U}^{n, s} \sin (n \omega t) \\
& \boldsymbol{f}(t)=\boldsymbol{F}^{0}+\sum_{n=1}^{H_{f}} \boldsymbol{F}^{n, c} \cos (n \omega t)+\boldsymbol{F}^{n, s} \sin (n \omega t)
\end{aligned}
$$

where $\omega$ denotes the frequency and $U^{(n, c, s)}, F^{(n, c, s)}$ the Fourier coefficients of the series.

Substituting Eqn.(7) into Eqn (5) and balancing the harmonic terms $H_{u}$ and $H_{f}$ leads to a nonlinear algebraic system with a size $n d o f_{u}\left(2 H_{u}+1\right)+n d o f_{f}\left(2 H_{f}+1\right)$. This equation system is then written in quadratic form:

$$
\boldsymbol{R}(\tilde{\boldsymbol{X}})=\boldsymbol{L}_{0}+\boldsymbol{L}(\tilde{\boldsymbol{X}})+\boldsymbol{Q}(\tilde{\boldsymbol{X}}, \tilde{\boldsymbol{X}})=\mathbf{0}
$$

where the nonlinear normal mode is characterised by the vector $\tilde{\boldsymbol{X}}$ (which contains the frequency $\omega$ ) and depends on the amplitude of the displacement. In this study the nonlinear normal mode is parametrized by the energy of the mode. A continuation method permits to build the frequency energy plot step by step.

The quadratic algebraic system Eqn (8) is solved with the ANM method which is based on a power series expansions of the unknowns $\tilde{\boldsymbol{X}}$ with respect to the path parameter $a$ [13], which is the arc-length of the Amplitude-Frequency curve of the nonlinear normal modes.

$$
\tilde{X}(a)=\tilde{X}_{0}+a \tilde{X}_{1}(a)+a^{2} \tilde{X}_{2}(a)+\ldots+a^{N} \tilde{X}_{N}(a)
$$

where $N$ is the order of the asymptotic numerical method. The value of this order is usually fixed between 10 and 20 .

The coefficients $\tilde{X}_{i}$ are calculated by recurrence [13] using the Jacobian matrix of the Eqn.( 8) and solving a linear system. The ANM has two advantages: it generates a succession of continuous branches, instead of a sequence of points to compute the NNM and the Jacobian matrix needs to be factorized only once by step (unlike a predictor-corrector method). This last property allows to use the method with finite element models, without the need of a model reduction, since once the Jacobian has been calculated no further computations are required. This method has been implemented by Moussi [11,14], which will be linked in the following to the polynomial chaos expansion to compute the uncertain nonlinear normal modes.

\section{NON INTRUSIVE POLYNOMIAL CHAOS EXPANSION WITH THE ANM}

The uncertainties propagation of NNMs for blade casing interaction is studied using the Polynomial Chaos Expansion (PCE) in a non-intrusive way. This requires to express the stochastic variable by an appropriately chosen polynomial to facilitate the calculations of expectations [15].

The goal is to reduce the infinite-dimensional random space to a finite-dimensional space that is feasible to compute. This is accomplished by parametrizing the random space with a finite number of mutually independent random variables. Let us consider a random variable $\xi$ defined in a random space $\Omega$ of dimension $d$, with a cumulative distribution function $F_{\xi}(\xi)$ and finite moments. Considering a random variable $\xi$ and a stochastic function $Y(\xi)$, the generalised polynomial chaos expansion $y$ (truncated at the order $\mathrm{P}$ ) is:

$$
y(\xi)=\sum_{k=0}^{P} \beta_{k} \Psi_{k}(\xi)
$$


where $\beta_{k}$ are the coefficients of the basis (or stochastic modes) that have to be calculated, while $\Psi_{k}(\xi)$ are the polynomial chaos basis functions. One considers the cumulative density function (CDF) such that $d F_{\xi}(\xi)=p(\xi) d \xi$, where $p(\xi)$ is the probability function (PDF) of the random variable $\xi$. The polynomial chaos basis functions for this distribution are the orthogonal polynomial functions satisfying:

$$
\int_{\Omega} \Psi_{n}(\xi) \Psi_{m}(\xi) p(\xi) d \xi=\gamma_{n} \delta_{m n}
$$

where $\Psi_{m}(\xi)$ are the orthogonal polynomials with a weight function (the PDF $p(\xi)$ ), $\gamma_{n}$ are constants and $\delta_{m n}$ is the Kronecker delta. This establishes a correspondence between the distribution of the random variable $\xi$ and the orthogonal polynomials of its PCE basis. Some of the correspondences between the PDF of $\xi$ and its PCE basis polynomials can be found in the literature $[15,16,17]$. Given the random space dimension $d$ and the maximal polynomial degree in the expansion $p$ (also called chaos degree), the number of terms in the Eqn.(10) is given by:

$$
P+1=\frac{(d+p) !}{d ! p !}
$$

The coefficients (stochastic modes), $\beta_{k}$, of the sum in Eqn.(10) can be calculated following different intrusive or non-intrusive approaches. In this paper a non-intrusive collocation method (presented in Fig.(2)) is proposed.

Non-Intrusive Collocation Methods (NICM) require the residue of the governing equations to be zero at discrete nodes in the computational domain. These nodes are called collocation points. The NICM consists in solving a finite number of deterministic problems obtained from the stochastic problem by replacing the random variables with deterministic values. The nodal set is typically formed by the nodes of a Gaussian quadrature rule (cubature rule in multidimensional space) [15]. For instance, the $N$ collocation points in a one-dimensional random space are the zeros of the $N^{\text {th }}$ degree polynomial of the basis.

The aim of this method is to conduct discrete projections by using the definition of orthogonality:

$$
\beta_{k}=\frac{<y(\xi), \Psi_{k}(\xi)>}{\gamma_{k}}
$$

where the symbol $<,>$ indicates the inner product defined in Eqn.(11), with respect to the PDF. The last relation means that the stochastic modes can be computed by solving integrals. The problem is that the expression $y(\xi)$ is unknown at this stage and

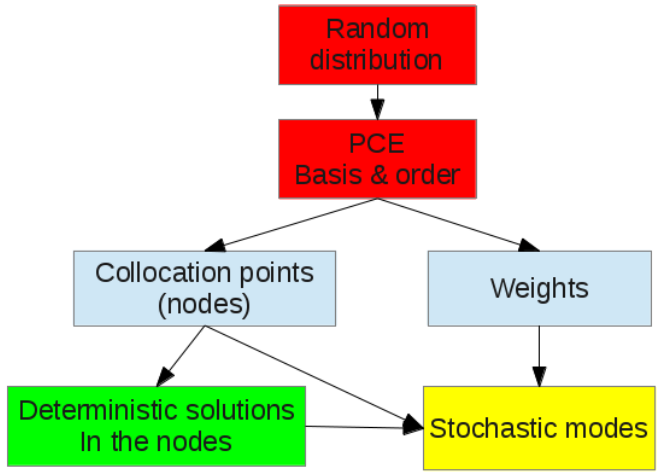

FIGURE 2. NON-INTRUSIVE COLLOCATION METHOD.

$\beta$ can not be computed explicitly. In order to overcome this problem the integrals are approximated using cubature formulas. Given a set of $N$ collocation points and the associated normalized weights (according to the PDF), after calculating $N$ deterministic solutions $y(\xi)$ Eqn.(13) becomes:

$$
\beta_{k}=\frac{1}{\gamma_{k}} \sum_{i=1}^{N} y\left(\xi_{i}\right) \Psi_{k}\left(\xi_{i}\right) w_{i}
$$

In this investigation the collocation solutions are the deterministic nonlinear normal modes characterized by the frequencyenergy plot (FEP). They are all computed with the ANM in a single run, allowing the calculation of the stochastic coefficient $\beta_{k}$ as a post treatment of the results from the ANM.

The Gaussian quadrature (14) can be adapted to multivariate random spaces $(d>1)$, i.e. Gaussian cubature $[15,16]$. One of the approaches to calculate the stochastic modes in the entire space is to use a tensor product rule. The problem thereby is that the number of collocation points increases exponentially with the dimension $d$. A significant reduction in the required collocation points can be achieved with the Smolyak sparse grids cubature [18].

After the calculation of the deterministic solution in $N$ collocation points, the stochastic modes by Gaussian quadrature (or Smolyak cubature in multivariate random spaces):

$\left(\begin{array}{c}<\Psi_{0}(\xi), \Psi_{0}(\xi)>\beta_{0} \\ \vdots \\ <\Psi_{P}(\xi), \Psi_{P}(\xi)>\beta_{P}\end{array}\right)=\left[\begin{array}{ccc}\Psi_{0}\left(\xi_{1}\right) & \ldots & \Psi_{0}\left(\xi_{N}\right) \\ \vdots & \vdots & \vdots \\ \Psi_{P}\left(\xi_{1}\right) & \ldots & \Psi_{P}\left(\xi_{N}\right)\end{array}\right]\left(\begin{array}{c}y\left(\xi_{1}, \frac{a_{1}}{A_{1}}\right) w_{1} \\ \vdots \\ y\left(\xi_{N}, \frac{a_{N}}{A_{N}}\right) w_{N}\end{array}\right)$

where $\frac{a_{i}}{A_{i}}$ are the arc length ratio, $w_{i}$ are the weights of the quadrature (or cubature) and $y$ represent the deterministic solution in the collocation points. 


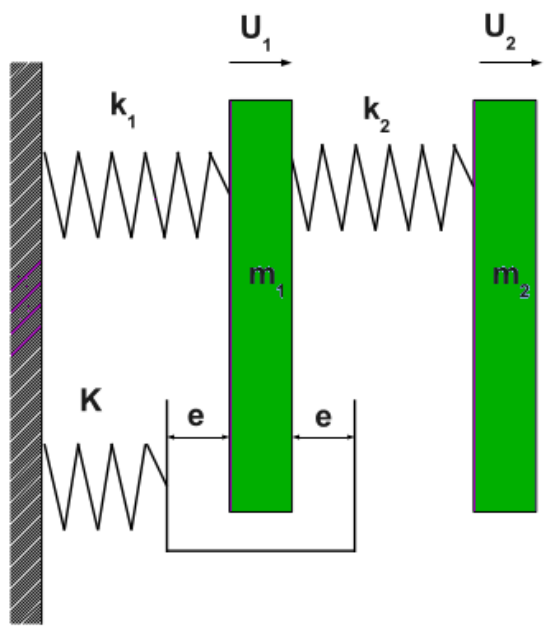

FIGURE 3. 2 DOFs model.

\section{NUMERICAL STUDIES}

The potential of the approach to combine the NNMs with nonintrusive PCE will be demonstrated with two numerical studies highlighting the influence of uncertainty on a nonlinear dynamic system with contact.

\section{Two degree of freedom system with gap}

A basic 2 DOFs model is studied first (see Fig. 3 ). A bilateral contact non-linearity is applied on the 1 st DOF with an uncertain clearance: $e=(1+0.2 \xi)$ where $\xi$ is a random uniform variable $\mathscr{U}(-1,1)$. The deterministic parameters of the model are: $m_{1}=$ $m_{2}=1 \mathrm{~kg}, k_{1}=k_{2}=1 \mathrm{~N} / \mathrm{m}, K=30 \mathrm{~N} / \mathrm{m}$.

The stochastic behavior due to the uncertain clearance is described on the energy-frequency plot. The PCE order was fixed to three, which permits a good accuracy. Initially the computation of the NNM is performed with a single harmonic. The resulting response plot of the first NNM can be seen in Fig.(4). At low vibration levels mass 1 is not contacting the end stops, and the uncertainty in the gap has no effect on the response. Once the amplitude is big enough to generate contact a smaller gap will lead to an early initiation of the nonlinear behaviour, whereas a wider gap will trigger it slightly later. It is interesting to see, that in the region of frequency change the influence of the uncertainty remains more or less the same, leading to a relatively narrow distribution band of the NNM curve of the first mode. Nevertheless the uncertainty of the gap can lead to frequency variations of up to $12 \%$ for a given energy level, highlighting the impact of the uncertainty on the system. Once enough energy is in the system to saturate the gap, the influence of the uncertainty once more recedes since the linear response dominates.

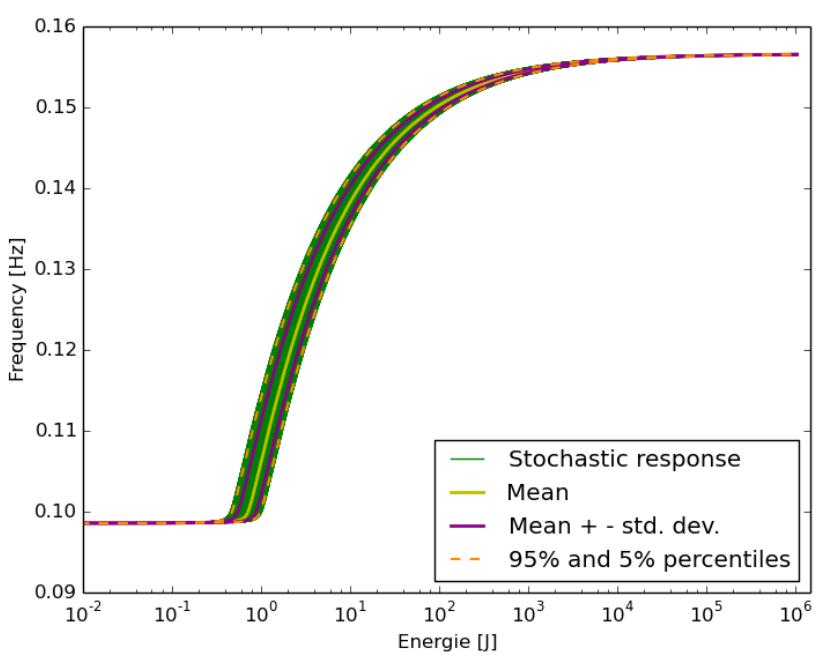

FIGURE 4. 2 DOFs model, stochastic NNM, 1st mode, 1 HARMONIC.

If the simulation is performed with 13 harmonics (which is necessary to describe accurately the nonlinear contact forces) two internal resonances appear on the energy-frequency plot as shown in Fig. (5 which seem to be leading to a saturation of the gap and a reduction of the impact of the uncertainties on the resulting NNM. Fig.(5) highlights the need to include the higher harmonics in the computation to identify the correct behaviour, and shows the effectiveness of the proposed approach to deal with uncertain internal resonances. Since this case is characterized by several returning points and strong discontinuities in the stochastic output, the Gibbs phenomenon [15] could distort the result and great care must be taken to avoid this from happening.

The stochastic 2nd NNM is shown in Fig.(6). In this case there is no internal resonance even when 13 harmonics are included in the computation. The uncertainty in the gap has a similar effect to mode 1 , but the uncertainty in the frequency range has increased to $50 \%$ during the closing of the gap.

The significant impact of the uncertainties on the NNMs of the simple 2DOF system highlights the potential of the proposed approach and confirms the need to take uncertainties into account when computing NNMs for a real structure.

\section{Blade casing interaction}

The impact of uncertainties on the NNMs of a real system was investigated for a blade casing interaction problem. A finite element model (819 degrees of freedom) of a turbine blade (Fig.(7)) with a localized unilateral contact has been studied with the introduced method. A non-linear gap element was applied at the 


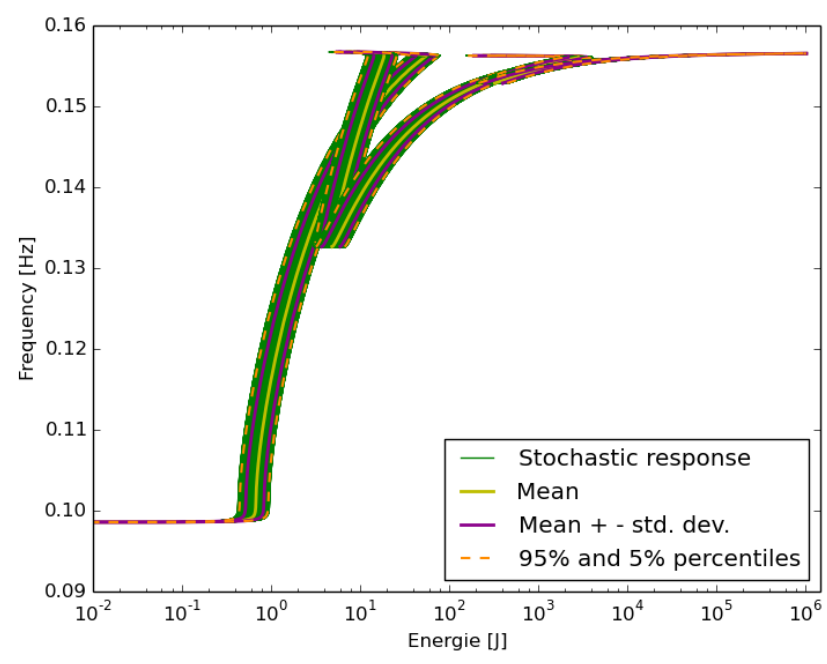

FIGURE 5. 2 DOFs model, stochastic NNM, 1st mode, 13 HARMONICS.

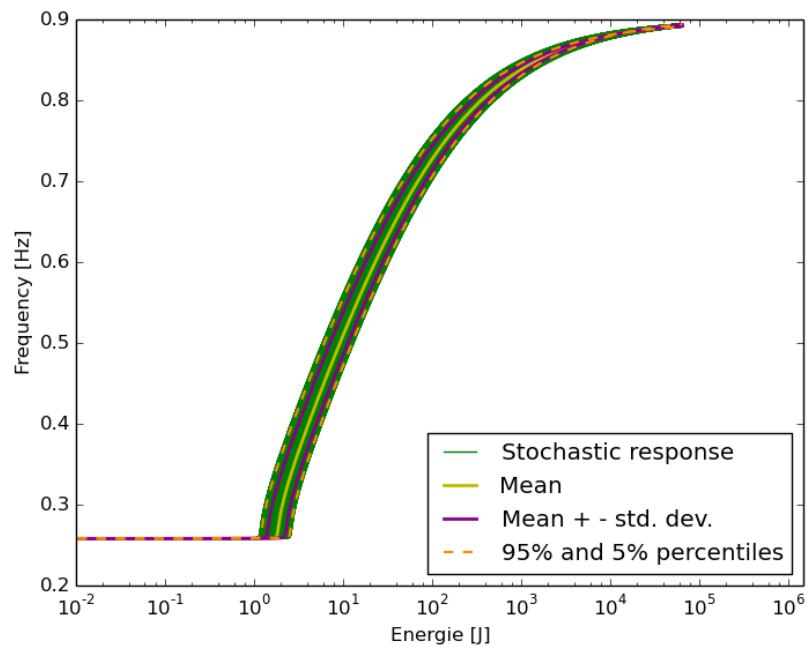

FIGURE 6. 2 DOFs model, stochastic NNM, 2nd mode, 13 HARMONICS.

trailing edge of the blade tip since the linear mode shapes of the blade indicated a likely contact in this location to the rigid casing.

The first four linear resonance frequencies of the blade and the presence of internal resonances (observed with the ANM) are listed in Table(1). Internal resonances due to the contact appear on the two considered bending modes. The internal resonances where during the building of the Frequency-Energy plot: for example an internal loop is observed on Fig.(8). In the case of the first bending mode the blade casing interaction excites the 1 st tor-

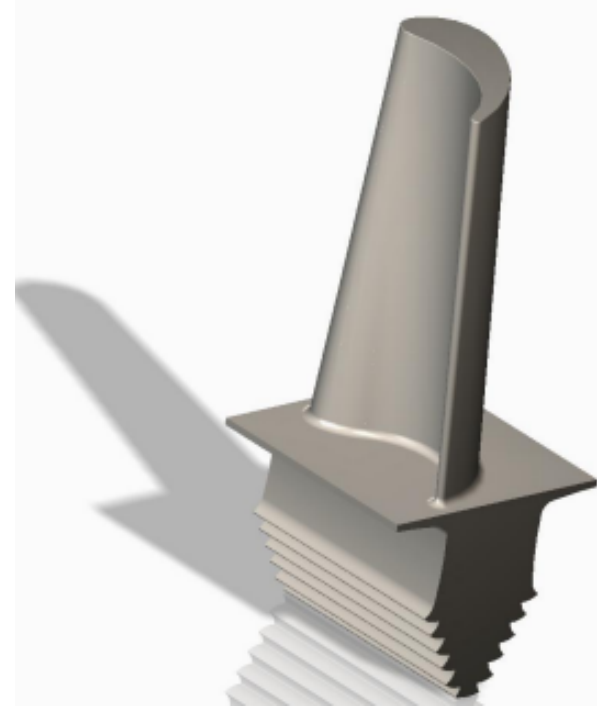

FIGURE 7. BLADE MODEL.

\begin{tabular}{|c|c|c|}
\hline MODE & FREQUENCY $[\mathrm{Hz}]$ (linear) & Internal resonance \\
\hline 1st (bending) & $2.99 \mathrm{e} 3$ & Yes \\
\hline 2nd (edgewise) & $4.94 \mathrm{e} 3$ & Not \\
\hline 3rd (torsion) & $9.05 \mathrm{e} 3$ & Not \\
\hline 4th (bending) & $14.37 \mathrm{e} 4$ & Yes \\
\hline
\end{tabular}

TABLE 1. FIRST FOUR MODES, BLADE.

sion mode as well, since at the level of the internal resonance the frequency of the third harmonic matches the natural frequency of the third mode (1st torsion mode). In Fig.(8) the deterministic 1st NNM is is calculated with 2 and 3 harmonics. With less than 3 harmonics the HBM-ANM behaves like a filter and the internal resonance cannot be captured. An additional interesting feature of the first NNM is the present of a bifurcation point (red dot in Fig.(8) ) which is caused by the existence of another solution branch starting at this point. For the computation of the full NNM it was necessary to build the curves in two steps: initially from a low energy level until the bifurcation point and then from a high level energy and decreasing the energy to reach the bifurcation point once more. Due to limitations of the implementation of the ANM in Code-Aster the bifurcation point could be detected, but it was not possible to trace the new branches from this point.

The initial PCE calculation (PCE order equals to 3) considered an uncertainty in the density and the Young's modulus of the blade with a Gaussian distribution for both parameters (with standard deviation of $1 \%$ ). These distribution and values were chosen arbitrarily but realistic in order to test the capability of the proposed method. Due to the size of the problem a Smolyak grid cubature technique [15] had to be used to reduce the number of collocation points to 17 for the PCE. The resulting uncertain of the first 


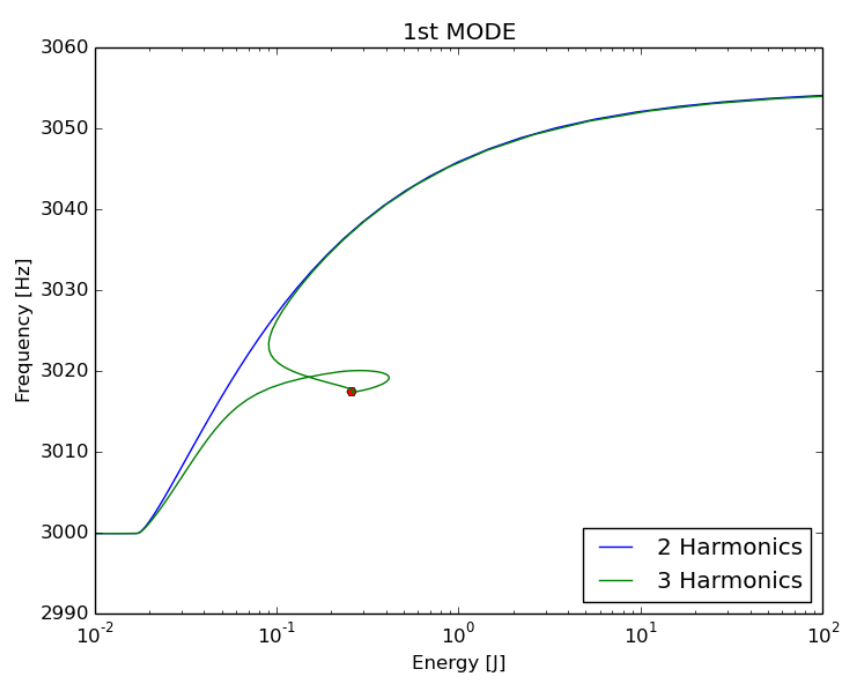

FIGURE 8. BLADE, DETERMINISTIC 1ST NNM.

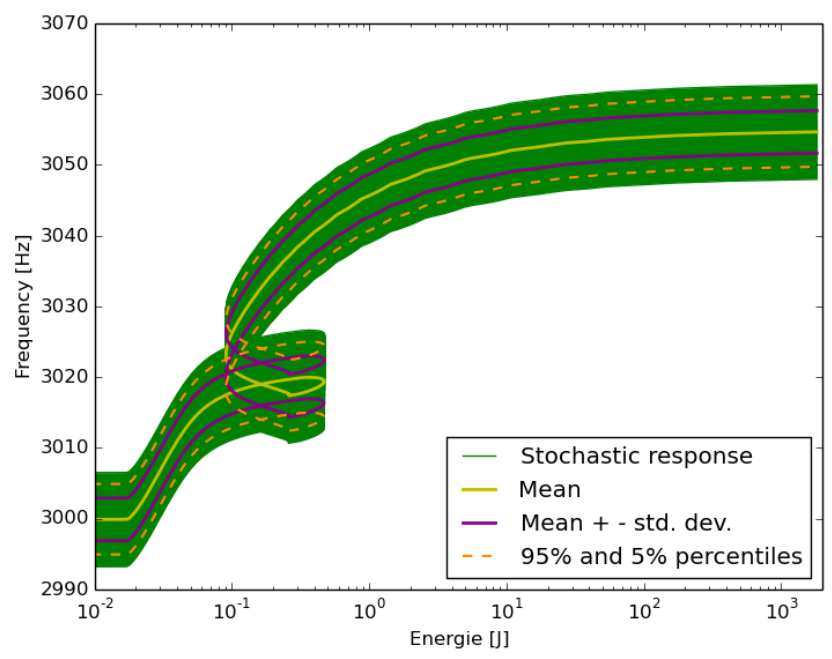

FIGURE 9. BLADE, 1ST CASE.

NNM for the blade casing interaction can be seen in Fig.(9). The stiffness of the system depends on the introduced uncertainties leading to an uncertain frequency for a given energy input in the $(0.5 \%)$. Due to the uncertain material properties, the entire NNM curve is impacted by the uncertainty. The internal resonance appears at the same energy level but due to the uncertainty in the system a much larger frequency shift can occur.

For the second blade casing study (Fig.(10)) the Young's modulus is considered as an Gaussian variable (with a standard deviation equals $7.5 \%$ ) while the clearance is defined by a Gaussian

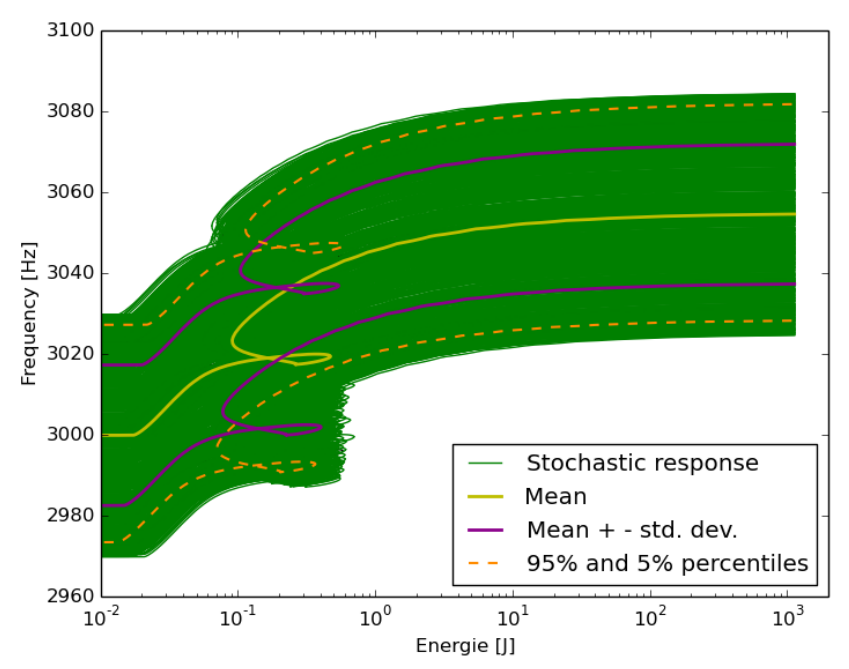

FIGURE 10. BLADE, 2ND CASE.

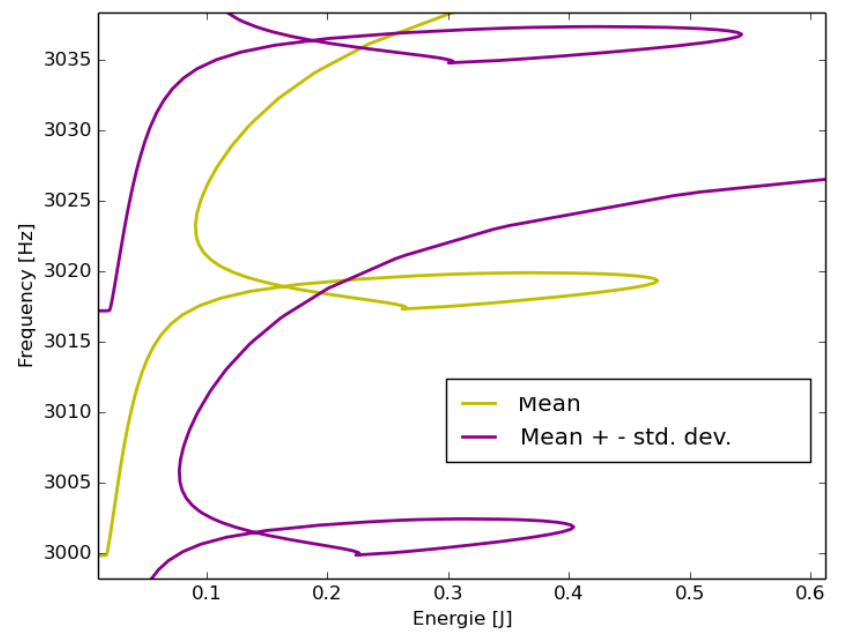

FIGURE 11. BLADE, 2ND CASE ZOOM.

random variable (with an amount of dispersion equal to $f 2 \%$ ). The PCE order is three. The Smolyak grid cubature technique is once more used with 17 collocation points. The stiffness of the system depends on the uncertainty on the Young's modulus and its effect on the energy-frequency plot is once more a frequency shift, also due to the larger variation of the Young's Modulus, E, the shift is significance stronger than in the previous case. The effect of the uncertain clearance can be seen in Fig.(11) where it can lead to shift in the energy levels at which the internal resonance is triggered and influences the maximum energy levels of the internal resonance. The impact of such an uncertain behavior 
on the response is important for the blade design, since the NNM can appear at unexpected energy levels, and may lead to significantly higher frequency shifts than predicted by the deterministic mode.

\section{CONCLUSION}

A new strategy has been proposed to calculate the nonlinear normal modes of uncertain systems with unilateral contact. The developed non-intrusive approach to calculate the stochastic coefficients for a polynomial chaos expansion allows using an existing code to compute the nonlinear normal modes (Frequency Energy Plot). The developed technique has been applied successfully to a 2DOF system and a blade casing interaction problem. It could be shown that the Coupling of the NNM with a HBM and ANM solution process, and the use of PCE allow a fast and reliable prediction of uncertain NNMs. The proposed method can deal with the dependence of the frequency on the energy in the system, and also works for the prediction of uncertain internal resonance. Uncertainties in the width of the nonlinear gap led to shift of the energy level at which the nonlinearity appears, and influenced on the maximum energy inside the internal resonance. Uncertainties in the Young's Modulus and density let to an uncertainty in the frequency level of the nonlinear mode. Future work will focus on robust design of blade shapes taking into blade-casing interaction and using the PCE to create a meta-model that is small enough for optimization loops.

\section{REFERENCES}

[1] Legrand, M., Batailly, A., Magnain, B., Cartraud, P., and Pierre, C., 2012. "Full three-dimensional investigation of structural contact interactions in turbomachines". Journal of Sound and Vibration, 331(11), pp. 2578-2601.

[2] Lesaffre, N., Sinou, J.-J., and Thouverez, F., 2006. "Model and stability analysis of a flexible bladed rotor". International Journal of Rotating Machinery, 2006.

[3] Padova, C., Barton, J., Dunn, M., and Manwaring, S., 2007. "Experimental results from controlled blade tip/shroud rubs at engine speed". Journal of Turbomachinery, 129(4), pp. 713-723.

[4] Almeida, P., Gibert, C., Thouverez, F., Leblanc, X., and Ousty, J.-P., 2014. "Experimental Analysis of Dynamic Interaction Between a Centrifugal Compressor and Its Casing". Journal of Turbomachinery, 137(3), Oct., p. 31008.

[5] Rosenberg, R., 1962. "The normal modes of nonlinear ndegree-of-freedom systems". Journal of applied Mechanics, 29(1), pp. 7-14.

[6] Jézéquel, L., and Lamarque, C.-H., 1991. “Analysis of non- linear dynamical systems by the normal form theory". Journal of sound and vibration, 149(3), pp. 429-459.

[7] Shaw, S., and Pierre, C., 1993. "Normal modes for nonlinear vibratory systems". Journal of sound and vibration, 164(1), pp. 85-124.

[8] Kerschen, G., Peeters, M., Golinval, J.-C., and Vakakis, A., 2009. "Nonlinear normal modes, part i: A useful framework for the structural dynamicist”. Mechanical Systems and Signal Processing, 23(1), pp. 170-194.

[9] Laxalde, D., and Legrand, M., 2011. "Nonlinear modal analysis of mechanical systems with frictionless contact interfaces". Computational Mechanics, 47(4), pp. 469-478.

[10] Didier, J., Sinou, J.-J., and Faverjon, B., 2013. "Nonlinear vibrations of a mechanical system with non-regular nonlinearities and uncertainties". Communications in Nonlinear Science and Numerical Simulation, 18(11), pp. 3250 3270.

[11] Moussi, E. H., Bellizzi, S., Cochelin, B., and Nistor, I., 2012. "Nonlinear normal modes of a two degree of freedom oscillator with a bilateral elastic stop". In $18^{\text {th }}$ Symposium Vibrations Chocs et Bruit.

[12] Cochelin, F. d. d., 1994. "A path-following technique via an asymptotic-numerical method". Computers \& Structures, 53(5), pp. $1181-1192$.

[13] Cochelin, B., and Vergez, C., 2009. "A high order purely frequency-based harmonic balance formulation for continuation of periodic solutions". Journal of Sound and Vibration, 324(12), pp. $243-262$.

[14] Code Aster documentation.

[15] Le Maître, O. P., and Knio, O. M., 2010. Spectral methods for uncertainty quantification : with applications to computational fluid dynamics. Series: Scientific computation. Springer, Dordrecht, Heidelberg, London.

[16] Xiu, D., and Karniadakis, G. E., 2002. "The Wiener-Askey Polynomial Chaos for Stochastic Differential Equations". SIAM J. Sci. Comput., 24(2), Feb., pp. 619-644.

[17] Brams, C., 2013. "Spectral methods for uncertainty quantification". Bsc. thesis, Technical University of Denmark.

[18] Kaarnioja, V., 2013. "Smolyak Quadrature". Master's thesis, University of Helsinki. 